\title{
Theodor Seifert (09.07.1931 bis 27.05.2018)
}

\section{Michael Ermann}

Online publiziert: 8. August 2018

(C) Springer Medizin Verlag GmbH, ein Teil von Springer Nature 2018

Als wir uns unter den Herausgebern dieser Zeitschrift einigten, ein Heft mit dem Schwerpunkt „Das Dunkle“ zu gestalten, kam mir sofort Theodor Seifert in den Sinn. Dieses Thema war wie für ihn geschneidert. Er musste über den „Schatten“ schreiben. Und er tat es auch.

Monate später saß ich über der Schlusskorrektur seines Textes. Da erreichte mich die Nachricht, dass Theo nach langer, würdevoll ertragener Krankheit verstorben war. Die Lektüre seines Beitrages „Gedanken über das Dunkle“ im vorangehenden Heft des Forum der Psychoanalyse ${ }^{1}$ ist wie eine letzte Begegnung mit ihm. Sein weit gespanntes Denken in der „Dynamik der Gegensätze“ berührt die Mythen der Antike ebenso wie die Dramen der Weltliteratur und die aktuelle Tagespolitik. Dabei gelangt er zu dem Schluss, ,,dass also das Böse, Dunkle seinen Sinn in der Erkenntnis hätte, das Nichtgute zu sein“.

Seifert gehörte seit der Gründung des Forum der Psychoanalyse zum Kreis der Herausgeber und blieb der Zeitschrift verbunden, als das ursprüngliche Herausgebergremium später zum Beirat wurde. Bei seiner Wahl ließen wir uns davon leiten, dass seine sehr autonome, jungianisch geprägte Auffassung des Unbewussten, die für Neuerungen und Randthemen wie die Mystik stets offen war, einen wichtigen Akzent in einem integrativen Gremium darstellen würde. Die Vision eines schulenübergreifend pluralistischen Forums wurde in den Folgejahren allerdings nur punktuell Wirklichkeit. Es entwickelte sich zu einem Organ, das die Mehrheitsorientierung der deutschsprachigen Psychoanalytiker am sog. Mainstream abbildet. So waren Beiträge aus jungianischer (oder individualpsychologischer) Sicht Ausnahmen. Sei-

1 Seifert T (2018) Gedanken über „,das Dunkle“. Forum Psychoanal 34:117-129.

Prof. Dr. M. Ermann ( $\varangle)$

Innsbrucker Str. 56, 10825 Berlin, Deutschland

E-Mail: michael.ermann@t-online.de 
ferts Stimme war nur selten hörbar ${ }^{2}$. Seine Funktion erfüllte er als beständiger und umsichtiger Ratgeber im Hintergrund.

Einige Ecksteine markieren seinen bewegten Lebensweg3: Geboren in Zwickau, 1949 Übersiedlung nach Westberlin, Studium der Psychologie und Graphologie, danach graphologischer Gutachter in Schweden. Von 1961 bis 1967 Ausbildung am C. G. Jung-Institut, Zürich, als „Schüler“ von Marie Louise von Franz. Danach Übersiedlung nach Stuttgart und Psychologe in verschiedenen Funktionen am Psychotherapeutischen Zentrum (Forschungsstelle und Klinik), später - als Psychologe! - stellvertretender Klinikleiter. Von 1972 bis 1979 Mitbegründer und bald Vorsitzender des C. G. Jung-Instituts Stuttgart. Von 1978 bis 1998 Mitglied der Leitung der Lindauer Psychotherapiewochen. Seit 1994 niedergelassen in psychoanalytischer Praxis in Wimsheim im Schwarzwald; 2017 Übersiedlung nach Augsburg, wo er nach 10 Jahren Auseinandersetzung mit seiner Krankheit gestorben ist. Unter seinen vielen Buchveröffentlichungen nenne ich nur die Lebensperspektiven der Psychologie - Wege, Schnittpunkte, Gegensätze (Walter, Olten 1981) - ein Buch, dass Einblicke in ,Erfahrungen mit einer gelebten analytischen Psychologie“ vermittelt.

Seifert war ein Mensch, der das Leben liebte und ausgekostet hat. Seine Vitalität, seine Ernsthaftigkeit und seine Wertschätzung der Eigenart des Anderen haben seine Beziehungen geprägt und Begegnungen mit ihm unvergessen werden lassen. Er war ein Mann für Freundschaften, der Verbindungen nicht abreißen ließ - so auch die zum Forum der Psychoanalyse. Dafür sind wir ihm dankbar.

\footnotetext{
${ }^{2}$ Seifert gestaltete den Schwerpunkt „Zur Aktualität der Jung'schen Analytischen Psychologie“ in Heft 2/2014.

3 Vgl. Seifert AL (2011) Theodor Seifert - Ein Leben im Spannungsfeld der Gegensätze. Und: Ahlert R (2011) Lieber Theo. Beides in: Müller L, Müller A (Hrsg) Der mittlere Weg zwischen Himmel und Erde. Festschrift für Theodor Seifert. Opus magnum, Stuttgart. Dort auch eine Sammlung bedeutender Aufsätze von T. Seifert.
} 